Musées, Patrimoine et Culture scientifiques et techniques

$114 \mid 2007$

novembre - décembre 2007

\title{
Des collections de minéraux pour quoi faire ?
}

Marie Jullion et Jean-Claude Boulliard

URL : http://journals.openedition.org/ocim/688

DOI : $10.4000 /$ ocim.688

ISSN : 2108-646X

Éditeur

OCIM

Édition imprimée

Date de publication : 1 novembre 2007

Pagination : 13-22

ISSN : 0994-1908

Référence électronique

Marie Jullion et Jean-Claude Boulliard, «Des collections de minéraux pour quoi faire ? », La Lettre de I'OCIM [En ligne], 114 | 2007, mis en ligne le 02 février 2011, consulté le 19 avril 2019. URL : http:// journals.openedition.org/ocim/688; DOI : 10.4000/ocim.688 


\section{L'Antiquité et le Moyen-Âge : un héritage livresque}

L'accumulation de minéraux que l'on pourrait considérer comme des embryons de collection est attestée dès l'Antiquité. Les trésors des rois de Pergame (283-133 av. J.-C.) ou des Ptolémée en Égypte comprenaient des minéraux. Plusieurs collections romaines nous sont connues grâce à Pline l'Ancien (23-79 av. J.-C.).

L'intérêt pour les minéraux est cependant bien limité. Le seul ouvrage marquant que nous a légué l'Antiquité est le Peri Lithon de Théophraste (entre 314 et 305 av. J.-C.) basé sur la Physique de son maître Aristote. Il sera repris par Pline l'Ancien. On trouve aussi des écrits dans des traités de pharmacopée, comme le De materia medica de Dioscoride (Ir siècle ap. J.-C.). Quelques philosophes, comme le stoïcien Sénèque (4-65 ap. J.-C.), développent également quelques idées sur les minéraux.

L'héritage de l'Antiquité, longtemps ignoré, réapparaît vers 1260 avec le De Mineralibus d'Albert le Grand (1193-1280). Entre le Peri Liton et le De Mineralibus, il ne manque pourtant pas de textes sur les pierres, mais ils procèdent d'un genre bien particulier, celui des lapidaires. Ce sont des catalogues de pierres plus ou moins connues (et parfois imaginaires) qui compilent toute une tradition livresque sur leurs vertus médicinales, magiques, symboliques voire chrétiennes. Les plus connus sont ceux de Marbode (10351123) et d'Hildegarde de Bingen (1098-1179).

La pensée du Moyen-Âge est marquée du sceau de la croyance, de la foi et de la tradition. À l'orée de la Renaissance, la connaissance des minéraux est livresque. L'idée de collectionner des minéraux est, historiquement parlant, plutôt récente et elle a été, géographiquement parlant, plutôt limitée.

\section{Les collections de la Renaissance au XVII siècle : découvertes, visions et miroirs du Monde}

L'histoire des collections de minéraux commence surtout au $\mathrm{XVI}^{\mathrm{e}}$ siècle. L'intérêt qui émerge alors pour une nature perçue comme généreuse, plus ou moins magique et merveilleuse conduit les savants de l'époque à des espoirs démesurés dans les bienfaits qu'elle serait susceptible d'offrir.

\section{Les collections d'ingénieurs}

Les découvertes des riches gisements d'argent dans les Monts Métallifères (Bohème), en Saxe et aux Amériques (Potosi en Bolivie par exemple) incitent des esprits curieux (que l'on désigne sous le terme d'ingénieurs de la Renaissance) à s'engager dans l'étude des minéraux. Ils ont un grand sens pratique. Certains de leurs ouvrages sont une discussion entre deux interlocuteurs (l'un pratique, l'autre théorique). Ils préconisent l'expérience et l'observation mais ne renoncent pas à recourir à l'alchimie et à Aristote lorsqu'ils se risquent à quelques justifications théoriques. Ce quilis rejettent ce sont les références prises comme des dogmes immuables qui musellent les observations pratiques.

Certains ingénieurs rassemblent des minéraux. Ces premières collections sont élaborées dans le cadre d'un travail scientifique ou d'une pratique professionnelle. Les spécimens sont souvent issus des mines alentours et servent de référence, de matériau pour l'étude de leurs propriétés et de support de cours pour apprendre à reconnaître les minerais utiles. Les collections de G. Agricola (1494-1555), J. Mathesius (1504-1565), J. Kentman (1518-1574) et B. Palissy (1510-1590) sont des exemples marquants de ces collections d'ingénieurs.

\section{Les droguiers}

La pharmacopée héritée du Moyen-Âge en Occident est basée essentiellement sur les simples. La présence des minéraux dans les droguiers est marginale : elle s'accroît sous l'influence de Paracelse (1493-1541).

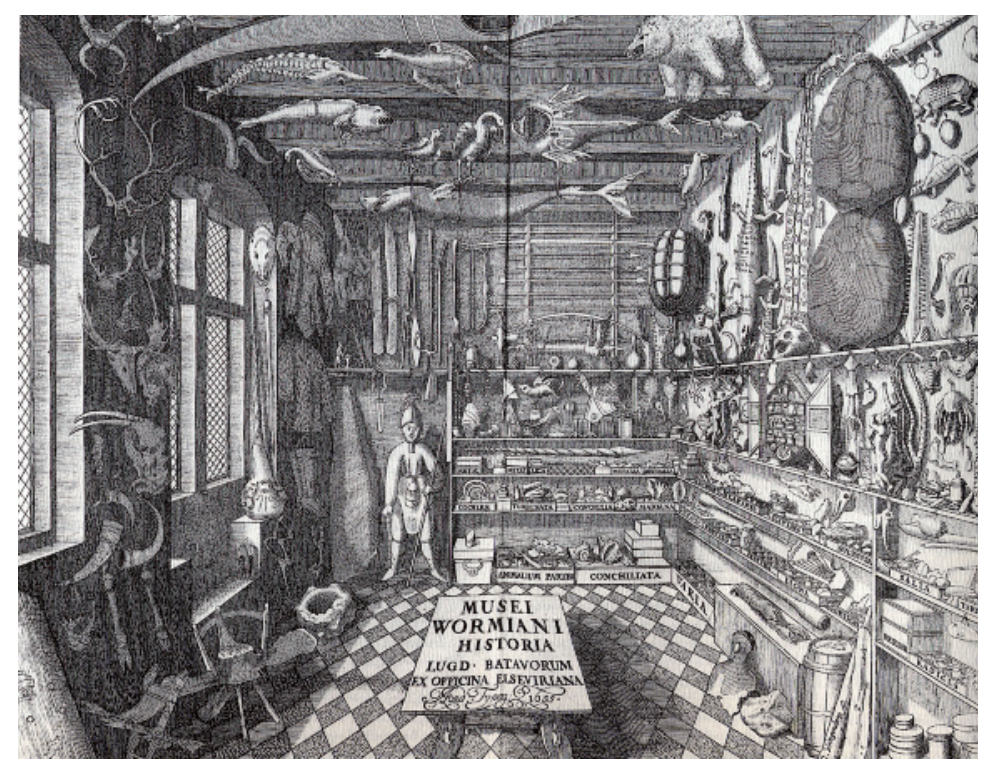

Musée Olao Worm : gravure tirée de Museum Wormianum, Leyde 1655. Le savant danois Olao Worm (1588-1654) avait constitué à partir de 1620 un cabinet qu'il a défini comme « une collection naturelle constituée avec la ferme intention d'amener vers la connaissance et loin de tout verbiage ». À sa mort, la collection s'est fondue dans le cabinet du roi Frédéric III où elle a perdu son identité. Elle a été reconstituée en 1988 
On lui doit d'avoir instauré l'usage systématique de minéraux et de sels métalliques comme médicaments. La plupart sont des poisons mais comme le dit Paracelse « tout est poison, rien n'est poison, seule la dose fait le poison ». Il préconisera l'utilisation des sels de mercure, d'arsenic, d'antimoine par exemple qui se développera jusqu'à la découverte des antibiotiques.

Le contenu des droguiers s'étend. De plus en plus ils contiennent des spécimens issus des trois règnes de la nature. Certains droguiers sont l'embryon de futures collections d'Histoire naturelle. En 1626, par exemple, le roi Louis XIII fonde à Paris le Jardin royal des plantes médicinales, un droguier à l'usage des droguistes et des pharmaciens. C'est la naissance de ce qui deviendra le Muséum national d'Histoire naturelle.

\section{Les Wunderkammer}

L'intérêt pour la nature se manifeste aussi dans ce que l'on appelle les Wunderkammer ou cabinets de curiosités. Ils se constituent dans l'esprit de permettre d'entrevoir dans un lieu restreint une vision, un «théâtre », un « miroir » du monde au travers de ses productions rares, précieuses, merveilleuses ou insoupçonnées. Ils sont une matérialisation de la vision du Monde et de la Nature si particulière de l'homme de la Renaissance que l'on a regroupé sous le terme de néoplatonisme de la Renaissance. L'alchimie y joue un grand rôle.

Ils peuvent réunir des naturalia (minéraux, animaux, plantes et fossiles), des artefacta (objets faits par l'Homme), des scientifica (instruments scientifiques) et des mirabilia (monstruosités de la nature). En ce qui concerne les productions du monde souterrain, les « pierres », on y trouve des minéraux, des roches, des minerais, des fossiles (dans le sens actuel), des «pierres »d'origine animale (comme les perles et les bézoards) voire des productions humaines alors ignorées (comme les silex taillés). On y trouve aussi des sculptures taillées dans des minéraux, les Handstein, formant une scène ou un paysage, le plus souvent minier. Certaines peuvent inclure des minéraux bruts. Pour la première fois, des minéraux naturels non travaillés et non transformés sont considérés comme précieux.

Quelques Wunderkammer appartenant à des puissants ou des monarques se distinguent par leur importance. Le but de ces collections que l'on peut qualifier de collections régaliennes, paraît particulièrement ambitieux : elles font partie du trésor et se doivent de participer au prestige du monarque, de montrer sa puissance et l'étendue de son savoir et de ses centres d'intérêts. À ce titre, elles sont le fruit d'une volonté personnelle voire d'une passion, que ne partageront pas nécessairement les successeurs intéressés à donner une autre image de leur règne. Certaines préfigurent les grandes institutions étatiques. Aux XVI ${ }^{\mathrm{e}}$ et $\mathrm{XVII}{ }^{\mathrm{e}}$ siècles, ces collections se multiplient en Europe. Parmi les plus célèbres on peut citer :

- la collection d'Auguste de Saxe (1526-1586) qui est à l'origine du musée de Minéralogie et de Géologie de Dresde,

- la collection de Ferdinand II du Tyrol (1529-1595), - la collection de l'empereur Rodolphe II (15521612) dont le « conservateur », le médecin A. Boèce de Boodt (1550-1632) a écrit la Gemmarum et Lapidum historia, qui a eu une influence durant tout le XVII ${ }^{\mathrm{e}}$ siècle et une partie du XVIII ${ }^{\mathrm{e}}$ siècle.

Ces grandes Wunderkammer posent des problèmes de rangement. En 1565, S. Quiccheberg (15291569), médecin personnel du duc Albrecht $V$ de Bavière, rédige le Inscriptiones vel Tituli Theatri amplissini qui est considéré comme le premier ouvrage de Muséologique connu. Il y catégorise soigneusement le contenu et définit ce que doit être l'organisation idéale d'un musée couvrant toutes les

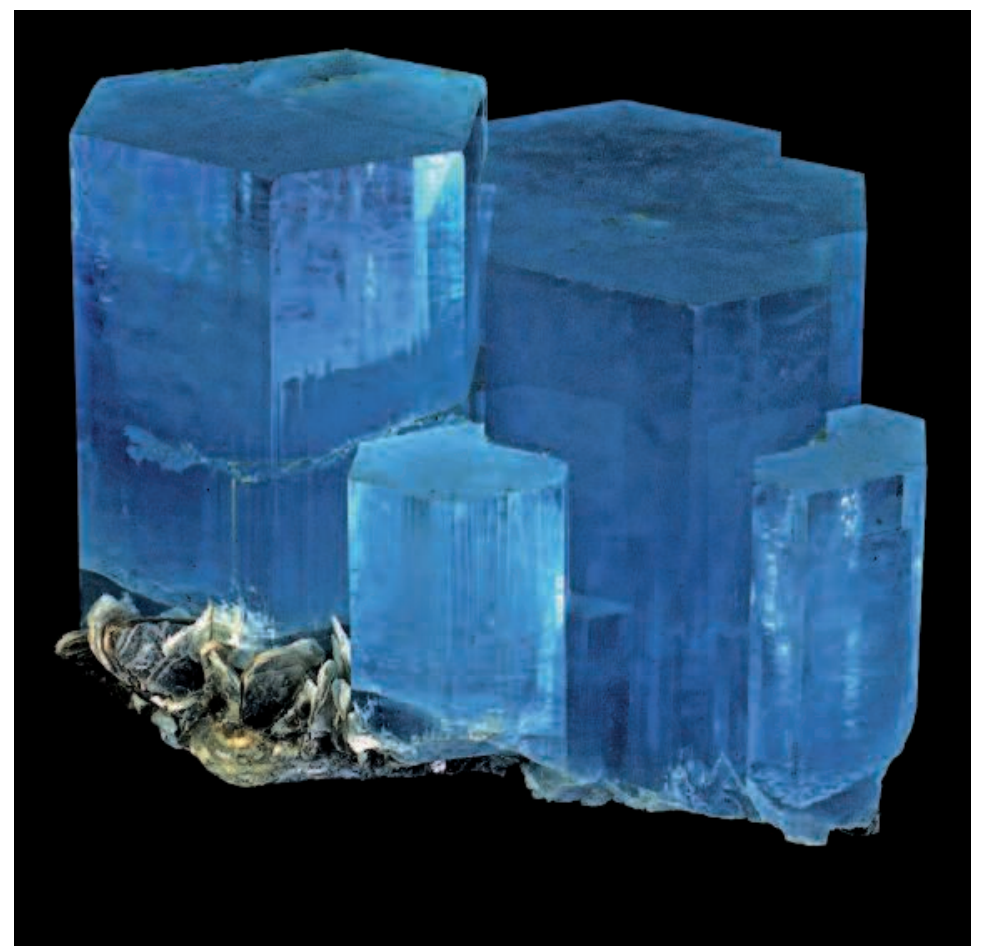

Béryl, variété aigue-marine $\left(\mathrm{Al}_{2} \mathrm{Be}_{3} \mathrm{Si}_{6} \mathrm{O}_{18}, \mathrm{Nagar}\right.$, Hunza val.,

$12 \times 11 \times 9 \mathrm{~cm}$, Northern Areas, Pakistan). Les gisements d'aigue-marine du nord du Pakistan sont connus depuis près d'un siècle, mais les spécimens de collection n'ont commencé à apparaître qu'au début des années 1980 . ๑) coll. de minéraux de l'université P. et M. Curie/J.P. Boisseau 
productions humaines et naturelles. Quiccheberg est le premier à employer le terme de Wunderkammer. Durant tout le $\mathrm{XVI}^{\mathrm{e}}$ siècle et une grande partie du XVII ${ }^{e}$ siècle, la vogue des Wunderkammer l'emporte sur celle de constituer des collections spécialisées : cette situation s'infléchit à peine à la fin de ce siècle.

\section{Les collections du XVIII" siècle : I'inventaire du Monde, le plaisir du beau monde}

\section{L'inventaire et les nomenclatures}

La science apporte au XVIII ${ }^{e}$ siècle ses Lumières qui relativisent beaucoup la vision néoplatonicienne de la Nature. Elles amènent un nouvel attrait pour l'industrie, l'artisanat, les sciences physiques et naturelles qui est concrétisé par le succès considérable de l'Encyclopédie de Diderot et d'Alembert. Les Wunderkammer déclinent. Les grands voyages scientifiques répondent à la volonté de récolter toutes les productions naturelles. Au désir de construire une représentation idéalisée de la Nature se substitue la volonté d'en établir un inventaire. Les collections d'alors peuvent être qualifiées d'encyclopédiques (dans la mesure où elles s'inscrivent dans la recherche d'une connaissance détaillée, encyclopédique, de toutes les productions naturelles).

La profusion de nouveaux spécimens demande un effort de classification énorme et plus compliqué qu'on ne l'imagine : la notion d'espèce minérale, par exemple, n'était pas établie et certains savants la contesteront jusqu'à la fin de ce siècle. On ne savait pas comment "ranger» les minéraux. Chaque minéralogiste d'envergure propose sa classification et sa nomenclature. Ces classifications ont participé à l'évolution de la science : la Cristallographie a pour origine le désir de Romé de l'Isle (1736-1790) de classer les minéraux selon leurs formes. Les travaux des nomenclateurs entrainent des réorganisations progressives. Les thèmes sont mieux segmentés peu à peu les minéraux, les roches et les fossiles (dans le sens moderne) sont séparés pour former des collections distinctes.

\section{Les minéraux :}

\section{un thème de collection à la mode}

La haute société s'entiche de Sciences naturelles et plus particulièrement de minéraux. Durant ce siècle, le nombre de collectionneurs privés de minéraux, souvent des aristocrates, explose : plus de 900 collectionneurs nous sont connus. La plus grande collection était celle de l'écossais J. Stuart (1713-1792), comte de Bute, qui contenait environ 100000 échantillons. Celle du prince hongrois J. Lobkowitz (1772-1816) contenait, roches et fossiles inclus, plus de 40000 spécimens pour un poids total estimé à 80 tonnes. Plus d'une vingtaine de collections dépassaient 10000 échantillons, plus d'une cinquantaine 4000 échantillons. À titre de comparaison, la collection de minéraux de Jussieu compte aujourd'hui 16500 échantillons environ.

La collection de minéraux connaît alors un âge d'or qui culmine à la fin de ce siècle : elle est la collection la plus prestigieuse en Histoire naturelle. Sa notoriété est comparable à celle des collections des Beaux-Arts et de Numismatique. Les résultats des ventes nous montrent que les prix de minéraux dépassent parfois ceux d'œuvres d'art prestigieuses : une pépite d'or ou une crocoïte surpassent un portrait de Rembrandt ou une sanguine de Michel Ange. Cette vogue touche tous les pays d'Europe, les États-Unis, le Mexique et le Brésil. Elle a participé à l'évolution de cette discipline. Des nomenclateurs, comme Romé de l'Isle, tiraient des revenus dans l'établissement de catalogues de collections.

\section{Vers les grandes institutions}

Les grandes collections de cour issues du XVII ${ }^{e}$ siècle se développent. D'autres grandes collections, qualifiées d'étatiques ou d'institutionnelles prennent naissance avec les premières académies des mines : Schemnitz en 1735, Potosi en 1756, Kongsberg en 1757, Prague en 1763, Freiberg en 1765, Berlin en $1770 \ldots$ Le nombre de collections accessibles au public augmente : il y en a près de 80 à la fin du XVIII siècle en Europe.

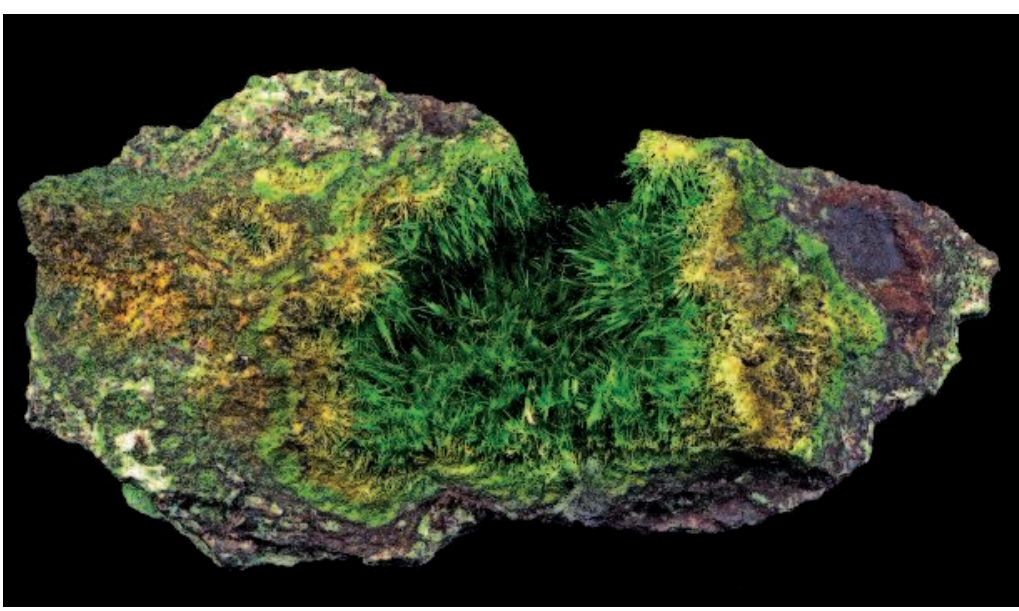

Cuprosklodowskite $\left(\mathrm{Cu}\left(\mathrm{UO}_{2}\right)_{2}\left(\mathrm{SiO}_{3} \mathrm{OH}\right)_{2} 6 \mathrm{H}_{2} \mathrm{O}\right.$,

$30 \times 15 \times 13 \mathrm{~cm}$, Musonoï, Katanga, Réublique démocratique du Congo). Ce minéral doit son nom au nom de jeune fille de Marie Curie, Sklodowska. (๑) coll. de minéraux de l'université P. et M. Curie/J.P. Boisseau 
En France les grandes institutions se développent, surtout à Paris. En 1729, Louis XV fonde le Cabinet d'Histoire naturelle du Roi. En 1793, la Convention réorganise le Jardin du roi et le nomme Muséum d'Histoire naturelle. Parmi les douze chaires, deux sont créées pour l'enseignement des sciences inorganiques : l'une de Minéralogie et l'autre de Géologie. En 1778, Sage (1740-1824) installe sa collection dans l'hôtel des Monnaies. Premier professeur de l'École des Mines de Paris, il s'en sert pour illustrer ses cours. Sa collection constitue l'embryon du cabinet de l'École des Mines (1783).

\section{Les collections du XIX siècle : le scientifique raisonnable et l'industriel déraisonnable}

\section{La science et ses raisons}

Au début du XIXe siècle, les progrès en Cristallographie et Chimie sont immenses, la Minéralogie moderne est née, elle devient une discipline très active dans beaucoup d'institutions. En France, en 1809, un décret impérial instaure une chaire de Minéralogie dans la nouvelle faculté des Sciences à la Sorbonne.

Cette science est de plus en plus complexe et de moins en moins accessible à l'amateur. Après la période fastueuse du XVIII ${ }^{e}$ siècle, elle devient, pour de nombreuses décennies, un thème de collection en stagnation chez les particuliers. Les collectionneurs ne disparaissent pas pour autant, mais ils sont différents. Ils sont souvent issus de la bourgeoisie plutôt que de la noblesse. Ce sont des amateurs éclairés, avides d'une science plus ou moins bien assimilée. La collection matérialise leur savoir. Le minéral n'a plus le statut d'objet précieux, il devient un symbole d'une science triomphante. Cet attrait de la science au détriment de l'esthétique est bien revendiqué dans la Minéralogie des gens du Monde, écrite par Pujoulx en 1813. On y lit que "les collections minéralogiques, si utiles pour l'étude, peuvent toujours se faire à peu de frais quand on n'a en vue que l'utilité et que l'on n'est point dirigé par un sentiment étranger à l'amour de la science... Quelques cabinets ne sont composés que de très beaux morceaux remarquables par leur grosseur ; ce sont des objets de parade et j'ai peu appris en voyant ces cabinets ».

Cet attrait est aussi perceptible dans les livres de Minéralogie de cette époque : contrairement à ceux de la fin du XVIII e siècle et du début du XIXe siècle, on n'y trouve presque plus d'illustrations de spécimens spectaculaires, ils sont remplacés par des schémas, souvent cristallographiques. Les anciennes collections privées du XVIII ${ }^{\mathrm{e}}$ siècle, connaissent des décotes terribles : la collection Lobkowitz par exemple est vendue en 1870 au tiers de ce qu'elle avait coûté. Par contre, les collections des grandes institutions s'accroissent et de nombreuses universités et écoles se dotent de collections qui sont des lieux d'étude et de science : les spécimens sont destinés à l'enseignement et à la recherche. Elles se spécialisent aussi suivant les centres d'intérêts des savants qui en ont la charge et les missions des institutions.

\section{L'essor de l'industrie minière :}

\section{un nouvel intérêt pour les minéraux}

La situation évolue à partir de 1860 environ. Une fois encore, comme au XVI ${ }^{e}$ siècle, c'est le développement d'une énorme industrie minière (charbon, or, diamant...) qui suscite un regain d'intérêt pour les minéraux. Les livres avec de belles illustrations refont leur apparition ( $\mathrm{La}$ Vie souterraine et Les Pierres de Simonin paraissent en 1867 et 1869). Ce renouveau prend sa source dans l'Est des États-Unis avec une nouvelle forme de collectionneurs, riches et sensibles au monde minéral qui a tant participé à la prospérité de leur nation. Ces collectionneurs ne s'intéressent qu'aux meilleurs spécimens connus,

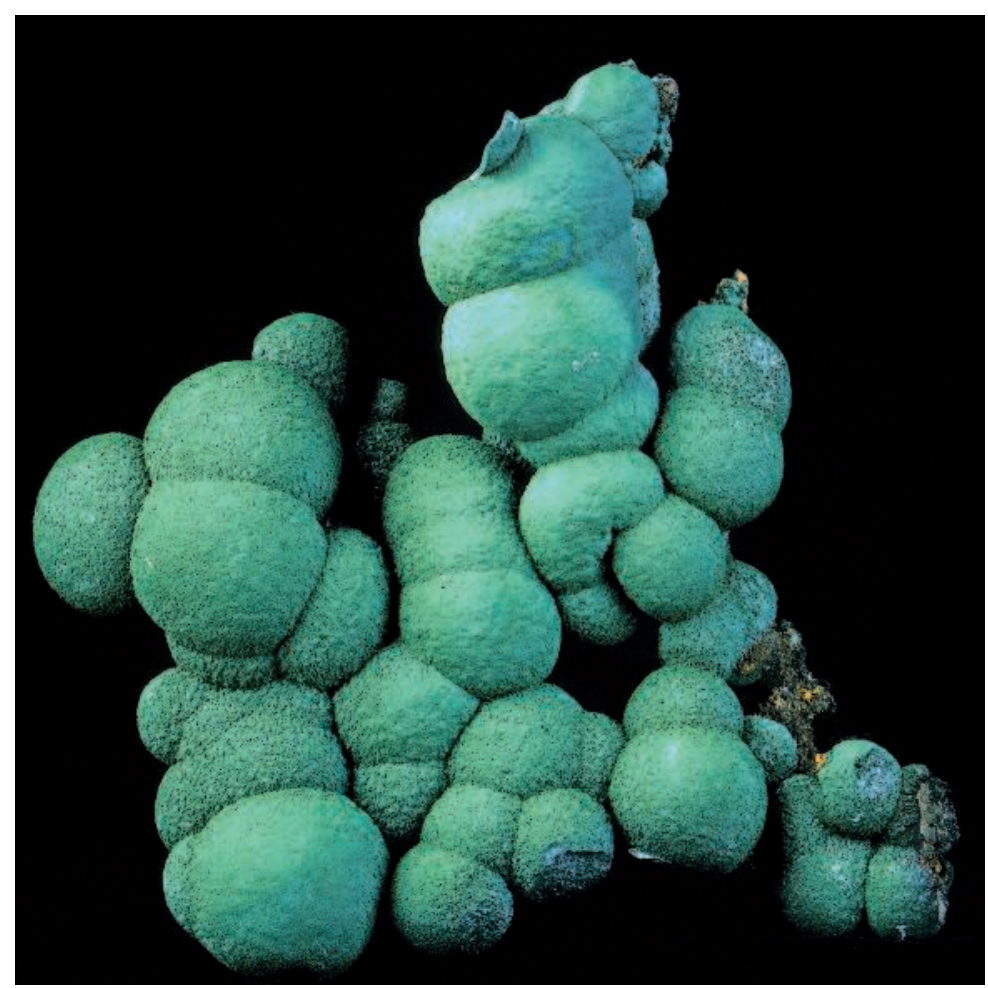

Malachite $\left(\mathrm{Cu}_{2}\left(\mathrm{CO}_{3}\right)(\mathrm{OH})_{2}, 16 \times 16 \times 5 \mathrm{~cm}\right.$, Kambove,

Katanga, République démocratique du Congo). La malachite, forme minérale du vert-de-gris, est utilisée en joaillerie pour sa couleur et les dessins en zones courbes ou concentriques de teintes différentes, révélées par le façonnage et le polissage. ๑) coll. de minéraux de l'université P. et M. Curie/J.P. Boisseau 
l'esthétique et la valeur vénale sont les critères importants. Ils n'hésitent pas à y mettre le prix, quel qu'il soit. Connaissant bien les affaires, ils considèrent que leurs collections ne représentent pas seulement leur richesse, leur savoir et leur pouvoir, elles sont aussi un investissement. Parmi eux, on trouve le magnat de l'acier, C. Bement (1843-1923) qui vendra sa collection, en 1901, au richissime financier J. Pierpont Morgan (1837-1913). On y trouve aussi W. A. Roebling (1837-1937) constructeur du pont de Brooklyn à New York. Hors des États-Unis, les grands collectionneurs sont rares et épars. En France, G. de La Bouglise, est connu pour sa collection d'ors natifs qui sera vendue en 1911 au magnat américain A. C. Burrage (1859-1931). Le colonel L. Vésignié (1870-1954) commence une collection qui le conduira au premier rang dans la première moitié $\mathrm{du} \mathrm{XX}^{\mathrm{e}}$ siècle. Les collections publiques ne sont pas non plus indifférentes aux minéraux spectaculaires, ce que confirme J.-P. Alibert (1820-1905), découvreur des gisements de graphite de Sibérie, lorsqu'il écrit, en 1901, que "la collection de la faculté des Sciences à la Sorbonne s'adresse aux savants et aux artistes ».

\section{Le $\mathrm{XX}$ siècle : l'abandon des scientifiques, une mauvaise période pour les collections}

La Première Guerre mondiale puis la crise de 1929 marquent l'arrêt de ce deuxième âge d'or. De 1920 à 1950 environ, les grands collectionneurs, comme le colonel Vésignié ou F. N. Ashcroft (1878-1949), sont devenus des «fossiles vivants ». Ils sont animés d'une véritable passion, rare et isolée, où la notion d'investissement est secondaire. Dès cette époque l'enseignement de la Minéralogie décroît dans les écoles et les universités. En 1983, P. Desautels (19201991), conservateur des minéraux de la Smithsonian Institution (Washington) résume ainsi la situation : «... les musées de minéraux ont été et sont encore malades. Mortellement malades. La science s'est déplacée et les a abandonnés ». Cette terrible sentence montre la fin d'une période, celle où les collections étaient surtout des lieux de science et de recherche. Ce déclin se traduit par l'arrêt ou la disparition de collections institutionnelles. Dans le meilleur des cas elles sont données à d'autres organismes : il en a été ainsi, à Paris, pour les collections de l'École Centrale, de la faculté de Pharmacie, du Collège de France et de l'Institut national d'Agronomie. Dans les cas moins favorables, elles sont dilapidées voire jetées. Cette hécatombe est importante jusque dans les années 1970-80, les exemples de disparitions deviennent rares à partir de l'an 2000. Ce mépris pour les minéraux se retrouve parfois au plus haut niveau de décision. Margaret Tatcher n'avait-elle pas dit que la collection du British Museum devait être jetée si elle ne valait rien ou vendue si elle valait quelque chose?

\section{La fin du $X X^{e}$ siècle : bouleversements et mutations, succès et périls}

Le développement des communications: les minéraux porteurs de message

Abandonnées des scientifiques, les grandes collections sont fragilisées. Comme lieux de transmission de la science, elles sont concurrencées par les centres de diffusion de la culture scientifique (CCSTI, centres d'interprétation...) qui prolifèrent en cette fin de siècle. Il ne s'agit plus ici de montrer des objets mais de communiquer. Avec le développement des moyens audiovisuels et informatiques, l'exposition « en masse » d'objets n'est plus nécessaire et seuls quelques objets sont présentés comme supports d'un scénario. Des grandes expositions deviennent « discursives » comme la Grande Galerie de l'Évolution à Paris pour le monde vivant ou l'exposition sur la Terre au Natural History Museum à Londres pour la Géologie.

Une autre évolution concerne les discours sur la science : il ne s'agit plus d'en faire l'apologie. À l'optimisme du XIX ${ }^{\mathrm{e}}$ siècle succède le pessimisme généré par les risques et les catastrophes scientifiques. La science pour la science se vend moins bien. La science étant critiquée, les collections n'en sont plus de facto une représentation, on veut de plus en plus en faire des supports de discours plus politiques, philosophiques, voire religieux (il existe au moins une exposition de "Sciences naturelles » créationiste aux États-Unis et naguère, on a échappé de peu à la réalisation, au Qatar, d'un muséum d'Histoire naturelle à vocation internationale récusant la théorie darwinienne). Cette évolution des collections «institutionnelles » de Sciences naturelles n'est pas systématique, surtout en Minéralogie. La cause principale est ce que l'on peut appeler le troisième âge d'or de la Minéralogie.

\section{Le développement du temps libre :} une nouvelle période faste

Desautels a oublié de dire dans son discours de 1983 que, dès les années 1960, est apparu un regain d'intérêt pour les minéraux. Il est bien mis en relief avec l'émergence et la multiplication des bourses de 
Minéralogie : la première a lieu à Tucson (Arizona) en 1955. En Europe, la première est celle de Zürich en 1960, suivie par celles de Sainte-Marie-aux-Mines en 1963 et de Munich en 1964. Cette période signe la naissance du troisième âge d'or de la Minéralogie, lié au développement de notre société de consommation et de loisirs où le tourisme prend de plus en plus d'importance. Le développement des mass medias et des communications sort les collections de leur cercle d'influence habituel. Toutes les franges de la société collectionnent. Desautels avait entrevu que les musées ont un rôle à jouer dans cette évolution. Loin de la critiquer, il décide d'en être un acteur car, écritil, " les grands collectionneurs sérieux perçoivent les musées comme une sorte de point d'ancrage dans ce marché de la collection ». L'engouement pour les minéraux s'accompagne d'une forte hausse des prix. Dans les années 1970 on pouvait avoir un spécimen de musée, hors matériaux précieux, pour quelques milliers d'euros. Au début du XXI ${ }^{\text {e }}$ siècle, pour le même type de spécimen il faut compter plusieurs dizaines à plusieurs centaines de milliers d'euros (le seuil du million a été franchi plusieurs fois).

Ce troisième âge d'or se traduit par une évolution forte dans notre société. Pour un public de plus en plus vaste, la vision des minéraux est renouvelée : de simples cailloux n'intéressant qu'un aréopage d'érudits ou de chercheurs spécialisés, ils sont devenus des objets remarquables et précieux. S'agit-il d'un effet de mode ou de quelque chose de plus profond ?

\section{Engovement passager ou transformation profonde ? Minéraux et patrimoine}

Pour y répondre il faut s'interroger sur la mise en patrimoine des minéraux. C'est une question qui n'a jamais été analysée alors qu'il s'est passé beaucoup de choses.

Il y a 40 ans environ, les conservateurs étaient plutôt favorables aux échanges. Le principal but était d'acquérir le plus grand nombre de spécimens. Les réserves étaient souvent alimentées par des missions sur le terrain. Le côté historique était souvent ignoré : des étiquettes anciennes ont été remplacées par des neuves, des collections léguées ont été dispersées dans la collection générale. L’idée dominante était que les minéraux, tout comme les autres objets de Sciences naturelles étaient renouvelables, à quelques exceptions près. La valeur (patrimoniale ?) d'une collection se mesurait au nombre et à la diversité de ses spécimens.

Il y a 30 ans, l'engouement par les amateurs devient de plus en plus sensible, les prix montent. Certains conservateurs le critiquent. En 1978 par exemple, Claude Guillemin (École des Mines de Paris) affirme que « les prix (...) que nous connaissons aujourd'hui, (...) me paraissent aussi ridicules que ceux qui acceptent de les payer ». Les découvertes de spécimens d'une qualité jusqu'alors insoupçonnée se multiplient. Les critères d'évaluation sont bouleversés et de nombreux spécimens anciens sont déclassés. De plus, des collectionneurs commencent à s'intéresser au côté historique et au pedigree des spécimens. Les missions sur le terrain sont de moins en moins rentables.

Il y a 20 ans, la plupart des collections se sont adaptées. La collection scientifique héritée du XIX ${ }^{\mathrm{e}}$ siècle décline. Plusieurs collections se replient sur leurs aspects historiques. D'autres, comme celle de Jussieu, de Los Angeles ou de Houston restent au plus près des nouvelles évolutions (environ 60 \% des spécimens exposés à Jussieu ont été acquis ces 40 dernières années). Les critères de choix dans l'acquisition sont une spécificité de la Minéralogie par rapport aux autres collections de Sciences naturelles. Elle est facile à comprendre. Pour le monde vivant (sauf la Paléontologie), il y a une correspondance directe entre le nom d'une espèce et son image. En Minéralogie, une espèce minérale renvoie souvent à un nombre considérable de formes et de matières.

Depuis 10 ans, il s'est établi un consensus sur l'évaluation d'une collection et sur ce que l'on connaît de meilleur : pour beaucoup d'espèces minérales, il y a

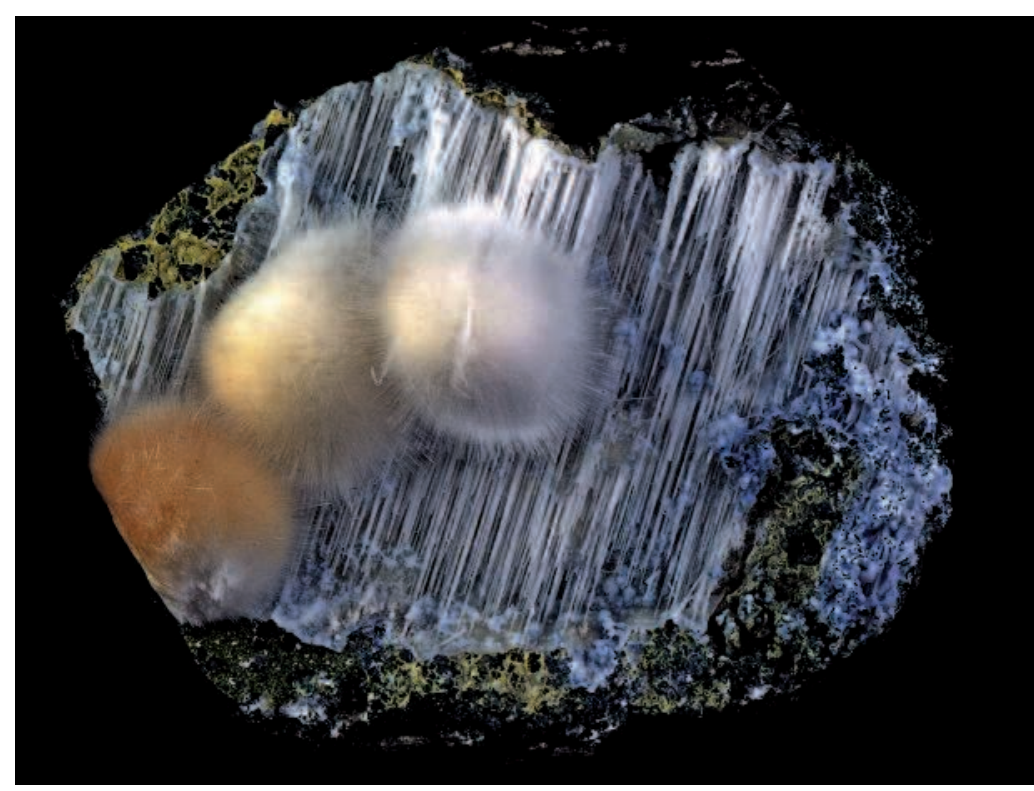

Mordenite $\left(\left(\mathrm{Na}_{2}, \mathrm{Ca}, \mathrm{K}_{2}\right)_{4}\left(\mathrm{AlSi}_{5} \mathrm{O}_{12}\right)_{8} 28 \mathrm{H}_{2} \mathrm{O}, 13 \times 11 \times 6 \mathrm{~cm}\right.$, Poona, Maharashtra, Inde). La mordenite est une zéolite qui se présente à Poona en boules blanches formées de cristaux très fins. ๑) coll. de minéraux de l'université P. et M. Curie/J.P. Boisseau 


\section{Bibliographie}

Babelon, J.-P. et Chastel A. La notion de patrimoine, Revue de l'Art, n ${ }^{49}$, 1980.

Boulliard, J.-C. Les collections de minéraux ? De l'utile à l'agréable, des tiroirs aux cimaises, Géologues, n¹40, 2004, pp. 65-68.

Boulliard, J.-C. et Geyssant, J. La collection des minéraux de Jussieu : retour d'expérience, Géologues, n¹40, 2004, pp. 84-86.

Collectif Des collections en sciences de la Terre pour quoi faire? Actes de la table ronde des 15 et 16 octobre 2002, OCIM et MNHN, 2005.

Desautel, P. et Chamberlain, S.-C. 9th Rochester Academy of Science mineralogical honors Paul E. Desautels, Rocks and Minerals, janvierfévrier 1983, pp. 5-11.

Gillman, B.-I. Museums Ideals of Purpose and Methods. Cambridge : Harvard University Press, 1923.
Guillemin, C. Évolution de la «minéralogie des gens du monde », Bulletin de la société française de Minéralogie, $\mathrm{n}^{\circ} 101$, 1978, pp. 124-132.

Jullion, M. Les collections de minéraux jusqu'à l'orée du XXe siècle : entre science et muséologie. L'exemple des trois grandes collections parisiennes, Mémoire de Master 2, Muséum national d’Histoire naturelle, 2005.

Pujoulx, J.-B. Minéralogie à l'usage des gens du monde. Paris, 1813.

Thomson, W.-A. Ikons, The Mineralogical Record, 2007.

Wilson ,W.-E. The History of mineral collecting, The Mineralogical Record, 1994

Wilson, W.-E., Bartsch, J.-A. et Mauthner, M. Masterpieces of the mineral world. Houston, 2004. 